\title{
Contributions of audition and vision to temporal rate perception
}

\author{
ROBERT B. WELCH and LANCE D. DUTTONHURT \\ University of Kansas, Lawrence, Kansas \\ and \\ DAVID H. WARREN \\ University of Califormia, Riverside, California
}

\begin{abstract}
Two experiments demonstrated that when both vision and audition are providing information about temporal rates in the range of 4 to $10 \mathrm{~Hz}$, audition has a much stronger influence on the bimodal percept than does vision. This case of auditory "dominance" over vision was shown to be neither the result of a difference between the sensory modalities in perceived intensity nor an artifact of the magnitude estimation procedure used by the subject to indicate perceived rate. It was concluded that these results provide support for a "modality appropriateness" hypothesis of the relative contribution of various sensory modalities in multimodal perception.
\end{abstract}

Characteristics of everyday objects are frequently perceived on the basis of information from several sensory modalities at the same time. Thus, one may simultaneously see and feel the shape of a pencil or see and hear the location of a barking dog. Of interest to the perceptual psychologist is the degree to which each of the involved sensory modalities contributes to such examples of multimodal perception. The nature of their relative contribution is, however, obscured by the redundancy of the sensory information. To disambiguate this situation, investigators have adopted the strategy of placing normally congruent sensory modalities into conflict with one another. The classic example is viewing the hand through an image-displacing wedge prism, thereby creating a discrepancy between the visually and proprioceptively perceived location of the limb (e.g., Hay, Pick, \& Ikeda, 1965). The rationale for this procedure is that by creating a discrepancy (typically, about $11^{\circ}$ ) between the two modalities it is possible to assess their individual contributions to the multimodal percept, since they are uniquely "tagged" by having different apparent locations. As shown by Hay et al. (1965) and many others (see Welch $\&$ Warren, 1980, for a review of this literature), the immediate outcome of viewing the prismatically displaced hand is to feel the limb to be located rather near where it is seen, a phenomenon referred to as "visual capture." More precisely, the felt position of the stationary hand is instantaneously shifted toward the displaced visual im-

\footnotetext{
The present investigation formed the basis of a paper delivered at the Meeting of the Psychonomic Society, San Diego, November 1983. The research was supported by a University of Kansas General Research Fund grant (No. 3407-0038). Reprint requests should be addressed to Robert B. Welch, Department of Psychology, University of Kansas, Lawrence, KS 66045.
}

age by about $70 \%$ of the imposed visual-proprioceptive discrepancy. The seen position of the hand is also affected, but to a much smaller extent; typically, it is biased toward the felt (true) position of the hand by about $30 \%$ of the intersensory discrepancy. ${ }^{1}$ The fact that the sum of the two forms of "intersensory bias" is approximately $100 \%$ indicates that a unified experience of the hand's location is achieved. A similar perceptual resolution occurs when vision is placed into spatial conflict with audition, an experience frequently referred to as the "ventriloquism effect"' (e.g., Howard \& Templeton, 1966, p. 361).

The phenomena of visual capture and ventriloquism strongly suggest that, under everyday circumstances of nondiscrepant perception, vision is much more heavily weighted than proprioception or audition when these modalities are providing redundant information about spatial location.

A reasonably well-supported explanation for the demonstrable dominance of vision in situations involving spatial localization is that vision is more heavily attended than are other simultaneously active spatial modalities (Canon, 1970, 1971; Kelso, Cook, Olson, \& Epstein, 1975; Uhlarik \& Canon, 1971; Warren \& Schmitt, 1978). This allotment of the observer's attention may, in turn, result from the fact that vision is a much better (i.e., more precise and accurate) modality for spatial perception than are the other so-called spatial modalities. This notion has been termed by Welch and Warren (1980) the "modality appropriateness" hypothesis.

The credibility of this hypothesis would be greatly enhanced if a task could be found for which vision was not the superior modality. Temporal rate would seem to be such a task, at least when comparing vision and audition, as seen in the fact that the visual critical flicker fusion threshold is substantially lower than the auditory flutter 
fusion threshold. This fact indicates, of course, that temporal acuity is greater for audition than for vision. Thus, it follows from the preceding arguments that when these two sensory modalities are providing information about temporal rate, the observer will attend more heavily to the auditory than to the visual stimulation, resulting in auditory dominance over vision. A number of previous studies provide support for this expectation. Gebhard and Mowbray (1959), Myers, Cotton, and Hilp (1981), and Shipley (1964) demonstrated that when the rate of a repetitive auditory stimulus (presented via earphones) is gradually increased or decreased, the perceived temporal rate of a flickering visual stimulus that is maintained at a physically constant level appears to change in the same manner. This phenomenon has been referred to as "auditory driving"' (Gebhard \& Mowbray, 1959). The reverse effect does not occur, however; changing the visual flicker rate does not alter perceived auditory rate. It may be deduced from these observations that if a fluttering sound and a flickering light are presented at somewhat different temporal rates, the physical discrepancy is unlikely to be perceived, but instead the two stimuli should seem to fluctuate together at approximately the perceived rate of the sound alone.

The auditory driving effect represents strong evidence that audition is the dominating modality in situations of visual-auditory temporal rate perception. There is, however, a methodological characteristic of the previous studies of this phenomenon that precludes an accurate assessment of the relative contribution of vision and audition to the percept. This is the fact that the procedure used for measuring auditory driving has always required the observer to attempt to match one of the two sensory modalities by means of the other. For example, in the study by Myers et al. (1981) subjects were instructed to set the rate of a fluttering sound so that it seemed to match a flickering light and vice versa. Clearly, it is not possible in this way to obtain a "pure" measure of the perception of temporal rate in either of the modalities, since the one used to indicate the observer's perception is itself affecting (and being affected by) the one being measured.

Two ways may be suggested for obtaining the desired undistorted measures of temporal rate for each of the modalities. One is to use some "outside"' modality, such as touch, assuming that this modality is demonstrated to be uninfluenced by either of the two primary modalities. The other possibility is to use a "modality-free" procedure. The latter alternative, in the form of direct magnitude estimation, was chosen in the present investigation.

The primary aim of the two experiments described here was to produce the auditory driving effect by means of the discrepancy procedure and to assess its strength in a manner uncontaminated by the effect itself. Also examined were the degree to which auditory dominance of visual temporal rate is influenced by the magnitude of the temporal rate, the spatial relationship between the auditory and visual stimuli, the direction and magnitude of the tem- poral rate discrepancy, and the intensity of the auditory stimulus. Finally, the present paradigm allowed for the detection and measurement of any biasing effect that vision might have on audition.

\section{GENERAL PROCEDURE}

In both experiments, the procedure for measuring auditory bias of vision and potential visual bias of audition was patterned after that of most previous studies in the area of intersensory bias effects (Welch \& Warren, 1980). Measured were: (1) perceived auditory rate alone, (2) perceived visual rate alone, (3) perceived auditory rate in the presence of a perceptually discrepant visual rate, and (4) perceived visual rate in the presence of a perceptually discrepant auditory rate. Measures (1) and (2) are referred to as the auditory and visual "control" measures, respectively; (3) and (4) are the "bisensory" measures. By means of these four measures, it is possible to calculate the individual contributions of vision and audition to temporal rate perception, using the following formulas:

Auditory Bias of Vision:

$$
[A(V)]=\frac{V-V_{a}}{V-A} \times 100
$$

Visual Bias of Audition:

$$
[V(A)]=\frac{A_{\nu}-A}{V-A} \times 100,
$$

where $V=$ apparent visual rate, $A=$ apparent auditory rate, $V_{a}$ $=$ apparent visual rate in the presence of discrepant auditory rate, and $A_{v}=$ apparent auditory rate in the presence of discrepant visual rate. Thus, for example, auditory bias of vision $[A(V)]$ will be $100 \%$ if the perceived visual rate, when in the presence of the discrepant auditory rate $\left(V_{a}\right)$, is the same as the perceived auditory rate alone $(A)$. It will be $0 \%$ if the perceived visual rate, when in the presence of the discrepant auditory rate $\left(V_{\alpha}\right)$, is the same as the perceived visual rate alone $(V)$. The same logic applies to visual bias of audition $[V(A)]$.

As indicated previously, intersensory bias is the perceptual response to an intersensory discrepancy. However, it happens that it is unnecessary to impose a discrepancy between visual and auditory temporal rates, since one already exists "naturally." That is, physically identical rates are not perceived as such, at least when measured separately (Myers et al., 1981). A 4-Hz auditory flutter, for example, is perceived to be slower than a $4-\mathrm{Hz}$ visual flicker when each is presented alone. ${ }^{2}$ The first experiment capitalized on this naturally occurring discrepancy between visual and auditory temporal rates.

\section{EXPERIMENT 1}

\section{Method}

Design. Subjects were exposed to repetitive visual and auditory stimuli, presented either alone (control trials) or simultaneously (bisensory trials) at rates of $4,6,8$, or $10 \mathrm{~Hz}$, and were required to indicate the perceived rate for each modality by means of direct magnitude estimation. A between-groups factor was the spatial relationship of the two stimulus objects: The visual stimulus was located straight ahead of the subject; the auditory stimulus (for separate groups) was placed $0^{\circ}, 45^{\circ}$, or $90^{\circ}$ to the right of straight ahead. In a fourth group, the sound was presented via earphones.

Subjects. Forty introductory psychology students ( 28 males and 12 females) at the University of Kansas served as subjects in the experiment as a means of fulfilling a course requirement. All had 
normal or corrected-to-normal vision and unimpaired hearing and were naive as to the purposes of the experiment.

Apparatus and Stimulus characteristics. The experiment took place in a dark $8.5 \times 12 \mathrm{ft}$ IAC anechoic chamber. The subject's chair included a restraint which served to maintain the head in the straight-ahead position. Visual temporal rates were presented via a standard $3 / 16$-in.-diam light-emitting diode (LED). For all four groups, the LED was located $6 \mathrm{ft}$ straight ahead of the subject, at eye level. The LED had a predominant frequency of $660 \mathrm{~nm}$ and an intensity of $1.5 \mathrm{mcd}$. Auditory temporal rates were presented via a 2 -in. loudspeaker. The speaker was located, for different groups, (1) straight ahead of the subject, 1 in. above the LED, (2) $45^{\circ}$ to the right of the LED, or (3) $90^{\circ}$ to the right of the LED. For the fourth group, the auditory stimulus was presented binaurally by means of standard earphones. The predominant frequency of the sound was $2.5 \mathrm{kHz}$, and its intensity, as measured at the subject's ear, was $52 \mathrm{~dB}$ SPL for the three ambient sound conditions and $68 \mathrm{~dB}$ SPL for the earphone condition. Temporal rates for both visual and auditory stimuli were produced by means of a digital square-wave oscillator located behind the subject. The "on time" for each auditory and visual pulse was $25 \mathrm{msec}$. The visual and auditory stimuli were presented at exactly the same rate and were in phase with each other.

Procedure. After a dark-adaptation period of approximately $20 \mathrm{~min}$, during which instructions were read to the subject and the visual and auditory stimuli were demonstrated, the vision-alone $(V)$ and audition-alone $(A)$ control measures were obtained. Each $V$ or $A$ control trial began with the presentation of a reference rate (in the same sensory modality) of $2 \mathrm{~Hz}$ for $3 \mathrm{sec}$, followed immediately by a faster rate, which varied from trial to trial in a nonsystematic fashion among the rates $4,6,8$, and $10 \mathrm{~Hz}$ and lasted for $10( \pm 2)$ pulses. There was a $15-\mathrm{sec}$ interval between each trial, during which the subject's response was recorded and equipment dials were changed in readiness for the next trial. The subject was instructed that the reference rate had a value of " 2 " and that his/her verbal estimate of the following rate should be made proportionate to the first (e.g., if the following rate appeared to the subject to be twice as rapid as the reference, he/she should respond with "four"). Thus, the subject was allowed to use any number greater than 2 to indicate his/her perception of the apparent rate of the light or sound. Four measures were obtained for each of the four frequencies for each modality. The mean of these four measures represented the value of the $A$ and $V$ scores used for the calculation of the subject's bias percentage. For half of the subjects, the 16 $A$ control measures were obtained first and the $16 V$ control measures second; for the other half, the order was reversed.

Following the control trials, the bisensory measures were obtained. Each bisensory trial began with a $2-\mathrm{Hz}$ visual-auditory temporal rate that lasted for $3 \mathrm{sec}$. The subject was informed that this rate had a value of " 2 ." This reference rate was followed immediately by a faster visual-auditory temporal rate, which varied nonsystematically among the four rates, $4,6,8$, or $10 \mathrm{~Hz}$, and lasted for $10( \pm 2)$ pulses. The subject was to assign (verbally) a number to the perceived auditory rate on half of these trials and a number to the perceived visual rate on the remainder of the trials. The experimenter's verbal cue, indicating to the subject to which of the two sensory aspects (auditory or visual) of the bisensory stimulus object he/she was to respond, was provided just before the onset of the bisensory stimulus. These two types of response, which represent the measures of $A_{v}$ and $V_{a}$, respectively (see previous formulas), were intermixed in a random manner, with a 15 -sec intertrial interval. The subject was cautioned not to ignore totally either the visual or the auditory aspect of the bisensory stimulus while estimating the rate of the aspect designated on a given trial. ${ }^{3} \mathrm{~A}$ total of four measures was obtained for each rate and for each modality. The mean of each of these two sets of four measures represented the value of $A_{v}$ and $V_{a}$ for a subject's bias percentage.

\section{Results}

The data from 6 subjects were discarded and replaced with those from 6 new subjects. Data were eliminated when a subject's percentage bias (when averaged across the four temporal rates) was found to be either less than $-50 \%$ or greater than $+150 \%$. This rejection criterion is essentially the same as that used in many previous studies of intersensory bias (e.g., Pick, Warren, \& Hay, 1969) and is based on the assumption that intersensory bias substantially greater than $100 \%$ or less than $0 \%$ is uninterpretable.

Examination of the results revealed that, across the different frequencies, the means and standard deviations of the $A, V, A_{v}$, and $V_{a}$ scores were positively correlated. Consequently, prior to the statistical analysis, a log transformation of each of the scores was performed in order to produce homogeneity of variance (which it did). Figure 1 depicts the $\log$-transformed $A, V, A_{v}$, and $V_{a}$ scores (multiplied by 10 ) as a function of temporal rate. The vertical distance between the $A$ and $V$ curves reveals the naturally occurring sensory discrepancy between physically identical auditory and visual temporal rates. (The difference between $A$ and $V$ curves for the untransformed scores was approximately $2 \mathrm{~Hz}$.)

Using the $A, V, A_{v}$, and $V_{a}$ scores (Figure 1), the two forms of intersensory bias, $A(V)$ and $V(A)$, were calculated by means of the formulas presented previously and are depicted as a function of temporal rate in Figure 2. A spatial locus $(4) \times$ temporal rate $(4) \times$ type of bias (2) analysis of variance, with repeated measures on the last two factors, was carried out. The only significant factor was type of bias $[F(1,36)=15.17, p<.001], A(V)$ being greater than $V(A)-52 \%$ and $10 \%$, respectively. Because temporal rate was not statistically significant, and because it entailed very few trials per rate, it was collapsed for the subsequent analyses.

Separate one-way analyses of variance, with spatial locus as the only factor, were performed on each of the two types of bias. The means of $A(V)$ and $V(A)$ were both significantly different from zero $[F(1,36)=72.11$, $p<.001$, and $F(1,36)=8.51, p<.01$, for $A(V)$ and $V(A)$, respectively], but in neither case was there an effect for spatial locus.

\section{Discussion}

By means of a response measure not involving a specific sensory modality (direct magnitude estimation), Experiment $\mathbf{1}$ demonstrated that the perceived temporal rate of a visual-auditory stimulus is determined primarily by audition, with a small, but reliable, contribution from vision. This dominance by audition is, of course, congruent with the "modality appropriateness" hypothesis, since, as discussed previously, hearing is demonstrably superior to vision in the perception of temporal rate.

Of interest was the finding that the degree of auditory bias of vision (and visual bias of audition) was essentially 


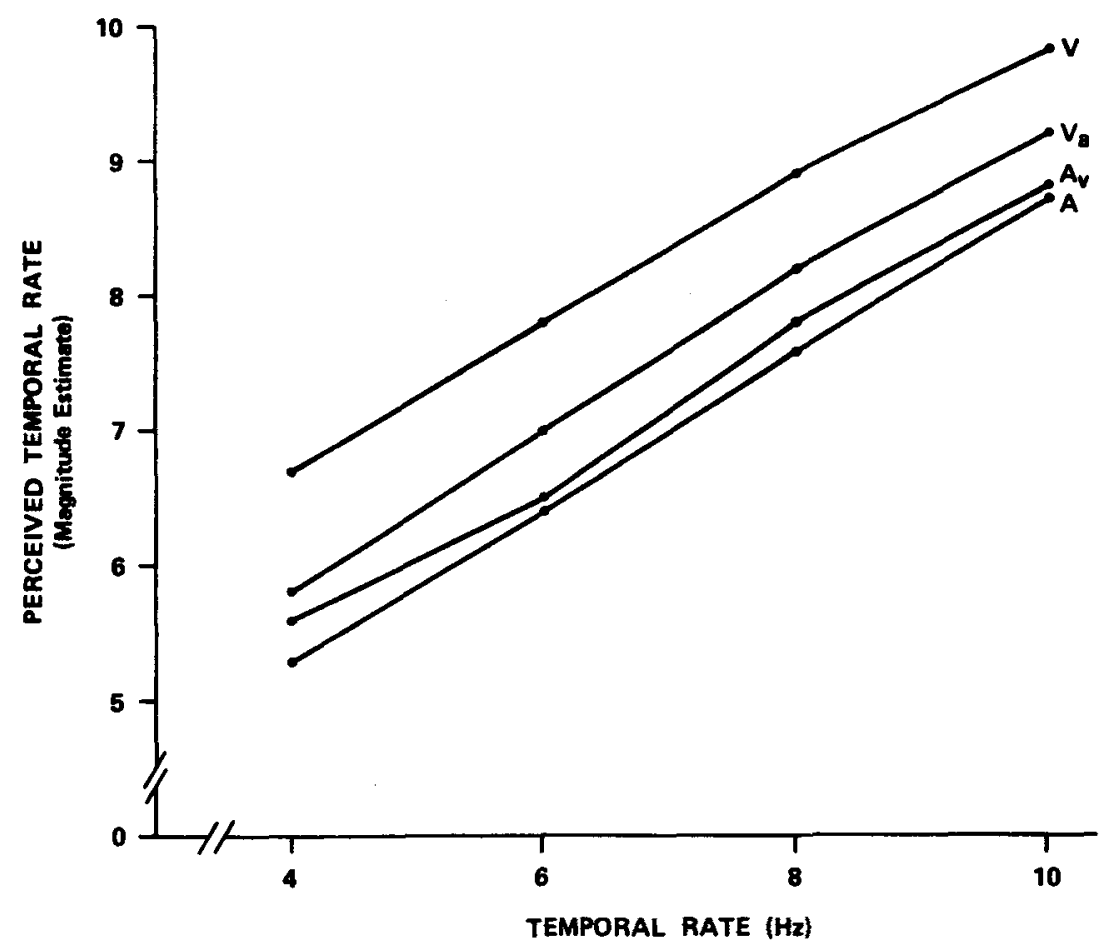

Figure 1. Experiment 1: Log-transformed "raw" magnitude estimates $(\times 10)$ for visual $(V)$ and auditory $(A)$ control measures and visual $\left(V_{a}\right)$ and auditory $\left(A_{v}\right)$ bisensory measures as a function of temporal rate (hertz).

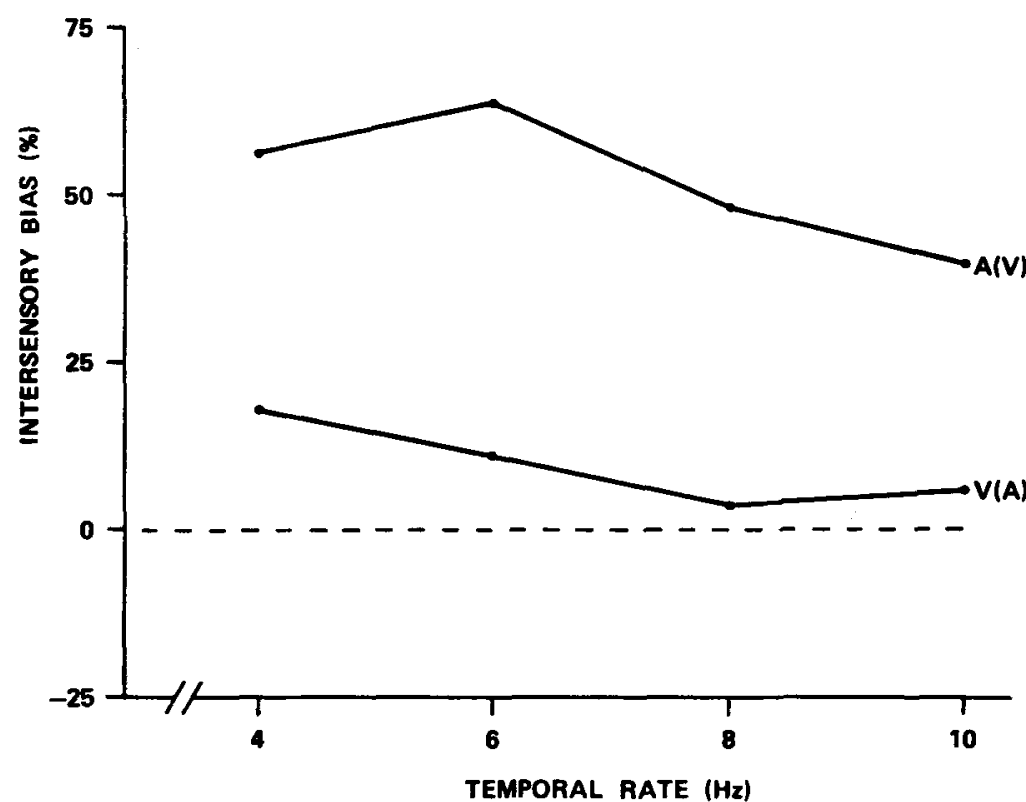

Figure 2. Experiment 1: Log-transformed percentage scores for auditory bias of vision $[A(V)]$ and visual bias of audition $[V(A)]$ as a function of temporal rate (hertz). 
the same, whether the visual and auditory stimuli were emanating from a single external spatial locus (straight ahead) or were separated by $45^{\circ}$ or $90^{\circ}$. This result contradicts the expectation, based on a model by Welch and Warren (1980) and previous literature (e.g., Warren \& Cleaves, 1971), that when the sound and light are in the same external location (presumably leading to a strong "assumption of unity") intersensory bias will be greater than when the two stimuli are spatially separated.

Although the results of the present study would appear to confirm the dominating effect of audition over vision in the perception of temporal rate, an alternative interpretation is possible. Specifically, it could be argued that perhaps what appears to be a strong auditory bias of vision is actually an artifact of the subjects' use of numbers in the magnitude estimation procedure. Thus, it is possible that, for reasons unknown, when subjects are confronted with the visual-auditory stimulus they respond by giving lower numbers for the perceived rate of the visual stimulus, but not because its rate is actually perceived to be lower. Thus, if this tendency exists, there would appear to be an auditory bias of vision when there is not.

Secondly, assuming that audition actually had a strong influence on visual perception in the first experiment, perhaps the reason was not because of the greater appropriateness of audition over vision for temporal rate perception, but because the sound was perceived to be more intense than the light. Experiment 2 dealt with the first of these issues by imposing discrepancies between the visual and auditory rates both in the direction of the naturally occurring one used in Experiment 1 (i.e., $V>A$ ) and, more importantly, in the opposite direction (i.e., $A>V$ ). Thus, for the $A>V$ group, for which perceived auditory rate was greater than perceived visual rate (when measured separately), true auditory dominance should cause the subject to ascribe larger numbers to perceived visual temporal rate, just the opposite of the postulated artifact. In order to deal with the possibility that the outcome of Experiment 1 was the result of subjects' perceiving the intensity of the auditory stimulus to be greater than that of the visual stimulus, the loudness of the sound was manipulated. The effect of the magnitude of the visualauditory discrepancy was also examined.

\section{EXPERIMENT 2}

\section{Method}

Design. Subjects were exposed to repetitive visual-auditory stimuli in which either the auditory rate was perceived to be 2,4 , or $6 \mathrm{~Hz}$ greater than the visual rate $(A>V$ group) or vice versa $(V>A$ group). The rationale for the choice of the visual-auditory combinations, which are presented in Table 1 , was the demonstration in Experiment 1 that a given $A$ control temporal rate (prior to the logtransformation) is perceived to be about $2 \mathrm{~Hz}$ slower than the same $V$ rate. On the basis of this information, it was thought, for example, that the pairing of a $2-\mathrm{Hz} V$ rate with $A$ rates of 6,8 , and $10 \mathrm{~Hz}$ (the first column of the $A>V$ group in Table 1) would result in intersensory discrepancies of approximately 2,4 , and $6 \mathrm{~Hz}$, respectively, with the auditory rate perceived as greater than the visual
Table 1

Pairings of Visual and Auditory Temporal Rates (in $\mathrm{Hz}$ ) Used in Experiment 2

$A>V$ Group

\begin{tabular}{lrrrrrr} 
& 10 & 12 & 14 & & \\
Auditory & 8 & 10 & 12 & & & \\
& 6 & 8 & 10 & & & \\
Visual & 2 & 4 & 6 & 6 & 8 & 10 \\
& & & & 6 & 8 & 10 \\
Auditory & & & & 4 & 6 & 8 \\
& & & & 2 & 4 & 6 \\
& & & & & \multicolumn{4}{c}{$V>A$ Group } \\
\hline
\end{tabular}

rate (when each modality is measured separately). Likewise, pairing a $4-\mathrm{Hz} V$ rate with $A$ rates of 8,10 , and $12 \mathrm{~Hz}$ (the second column of the $A>V$ group in Table 1) could be expected to produce discrepancies of approximately 2,4 , and $6 \mathrm{~Hz}$, respectively. For the $V>A$ group (the right half of Table 1), a $6-\mathrm{Hz} V$ rate paired with $A$ rates of 6,4 , and $2 \mathrm{~Hz}$ (the first column of the $V>A$ group in Table 1) should produce discrepancies of 2,4 , and $6 \mathrm{~Hz}$, respectively, with the visual rate perceived as greater than the auditory rate. Whether or not the $A-V$ discrepancies actually perceived by subjects were precisely as predicted was not important. Rather, what was crucial for the present argument was that each group produce results indicative of strong auditory bias of vision, which is to say that for the $V>A$ group the estimates of $V$ rates during the bisensory trials should be decreased relative to estimates of $V$ rates alone, whereas estimates of bisensory $V$ rates in the $A>V$ group should be increased. The second between-groups factor was the intensity of the sound; for half of the subjects, auditory intensity was set at $40 \mathrm{~dB}$, and for the other half, it was set at $52 \mathrm{~dB}$, a difference in loudness which subjects reported to be quite substantial.

Subjects. Forty subjects ( 23 males and 17 females) were obtained from the same population as in Experiment 1. None of the subjects had participated in the first experiment.

Apparatus and Stimulus characteristics. The apparatus and stimuli were the same as in Experiment 1, with the following exceptions. For all subjects, the 2 -in. loudspeaker was located 1 in. above the LED; both were positioned $6 \mathrm{ft}$ straight ahead, at eye level. The intensity of the sound, as measured at the subject's ear, was $40 \mathrm{~dB}$ SPL for half the subjects and $52 \mathrm{~dB}$ SPL for the remainder.

Procedure. After the 20-min dark-adaptation period, the subject made direct magnitude estimates of the visual and auditory rates that would later be paired with each other during the bisensory trials. Three control measures were obtained for each of the $A$ and $V$ stimuli (see Table 1). As in Experiment 1, each of these control trials began with a $3-\mathrm{sec}$ presentation of the $2-\mathrm{Hz}$ reference rate in the appropriate sensory modality. Unlike the previous experiment, three more $V$ and $A$ control measures were obtained after the bisensory trials. This was done as a check for the possibility that exposure to the bisensory stimuli produces a cumulative adaptation of one modality to the other, thereby distorting the values of percentage bias. (No evidence for such an adaptive shift between pre- and postbisensory measures was found.) Thus, the $A$ and $V$ values used in the bias calculation were derived by averaging the three prebisensory and three postbisensory trials control measures.

The bisensory measures were obtained in exactly the same fashion as in Experiment 1, the $2-\mathrm{Hz}$ bisensory reference rate being followed immediately by the (discrepant) bisensory rate, to which the subject was to respond either in terms of the apparent visual rate $\left(V_{a}\right)$ or the apparent auditory rate $\left(A_{v}\right)$. Each of these two types of bisensory trial occurred three times for each of the three temporal rate discrepancies $(2,4$, and $6 \mathrm{~Hz})$ at each of the arrays of temporal rates (see Table 1), for a total of 54 trials. 


\section{Results}

The data for 12 subjects were discarded and replaced, using the same rejection criterion as in Experiment 1. The majority of these subjects were lost to the experiment due to extreme bias scores $(>+150 \%$ or $<-50 \%)$ due primarily to unusually small $A-V$ denominators.

An examination of the $A, V, A_{v}$, and $V_{a}$ " raw" scores (prior to $\log$ transformation) indicated that the actual perceived discrepancies between $A$ and $V$ were approximately $1,2.5$, and $4 \mathrm{~Hz}$ for the $A>V$ group and $1.75,3.5$, and $5 \mathrm{~Hz}$ for the $V>A$ group, values somewhat less than those expected.

The mean log percentage bias scores are depicted in Figure 3, with the results for the $A>V$ group shown in the upper half of the figure and those for the $V>A$ group shown in the lower half. A direction of discrepancy (2) $X$ intensity of sound (2) $\times$ magnitude of discrepancy (3)
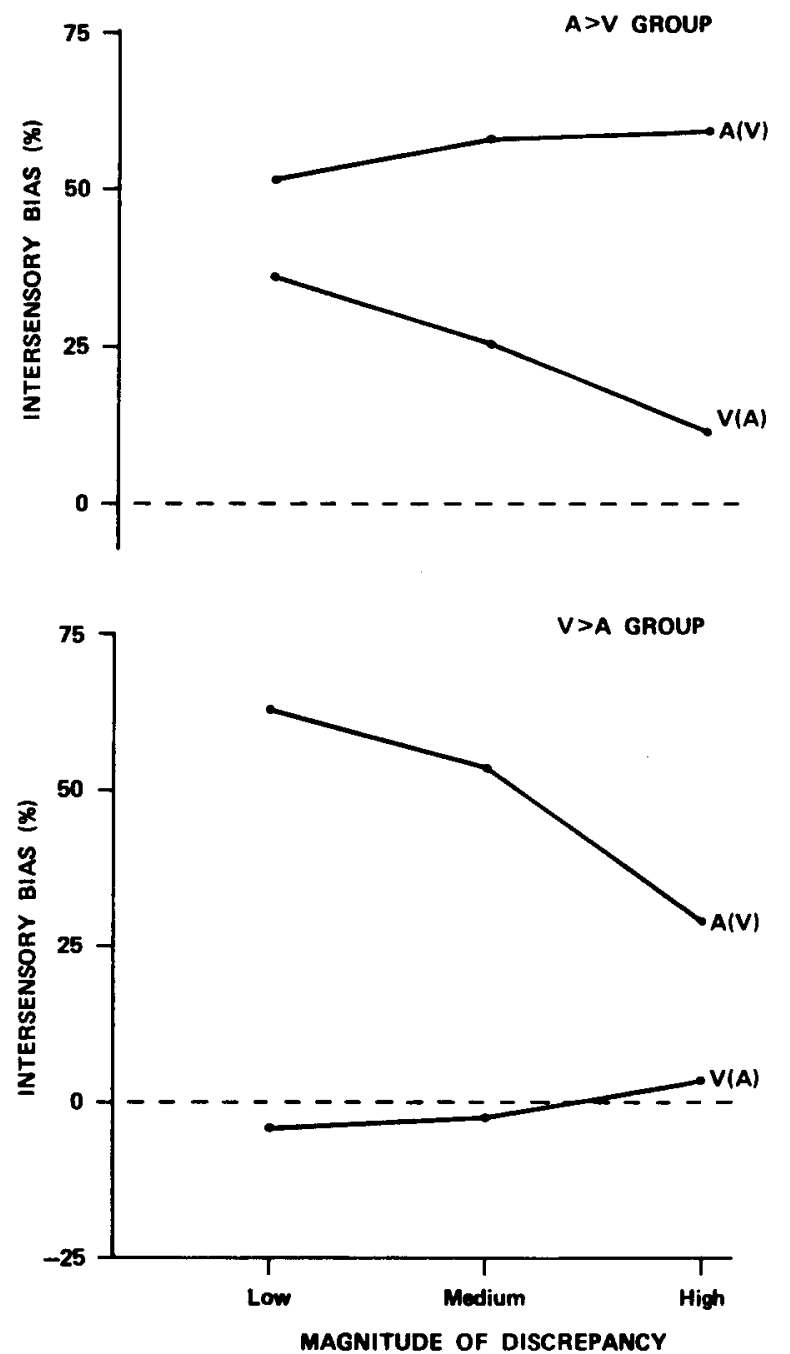

Figure 3. Experiment 2: Log-transformed percentage scores for auditory bias of vision $[A(V)]$ and visual bias of audition $[V(A)]$ for $A>V$ and $V>A$ groups as a function of magnitude of visualauditory discrepancy. analysis of variance was carried out separately for $A(V)$ and $V(A)$. These analyses revealed that both types of bias were significantly different from zero $[F(1,36)=118.74$, $p<.001$, and $F(1,36)=10.13, p<.004$, for $A(V)$ and $V(A)$, respectively]. The average $A(V)$, collapsed across the three visual-auditory discrepancies and both directions of discrepancy, was approximately $52 \%$; the average $V(A)$ was approximately $13 \%$.

It may be seen from Table 1 that the difference in direction of discrepancy between the $A>V$ and $V>A$ groups is confounded by the array of temporal rates used. Because interpreting the outcome of this comparison is therefore problematic, the subsequent analyses were performed for the $A>V$ and $V>A$ groups separately. According to an intensity of sound (2) $\times$ magnitude of discrepancy (3) $\times$ type of bias (2) analysis of variance for the $A>V$ group (upper half of Figure 3), there was a main effect for type of bias $[F(1,18)=8.48, p<.01]$, $A(V)$ being greater than $V(A)$, but none for intensity of sound $[F(1,18)=2.38, p>.05]$. The apparent magnitude of the discrepancy $\times$ type of bias interaction was not significant $[F(2,36)=1.99, p>.05]$.

The same analysis for the results of the $V>A$ group (lower half of Figure 3 ) revealed a main effect for both type of bias $[F(1,18)=19.54, p<.001]$ and magnitude of discrepancy $[F(2,36)=3.28, p<.05]$, but once again no main effect for intensity of sound $[F(1,18)=.58$, $p>.05]$. The magnitude of the discrepancy $\times$ type of bias interaction was significant $[F(2,36)=3.75$, $p<.05]$. An examination of the lower half of Figure 3 reveals a decline in $A(V)$ with increasing rate discrepancy but no change in $V(A)$.

\section{Discussion}

Experiment 2 produced clear evidence of auditory bias of vision, together with a small amount of visual bias of audition. Thus, even when the auditory temporal rate was perceptually greater than the visual rate (the opposite of the relation in Experiment 1), audition dominated vision. Furthermore, the absence of an effect for the loudness of the sound argues against the suggestion that the reason audition so strongly influences vision in the perception of temporal rate is that it is experienced as being more intense than the light. This finding, then, renders more likely the "modality appropriateness" explanation for auditory dominance in visual-auditory temporal rate perception.

\section{CONCLUSIONS}

The present investigation has demonstrated that when vision and audition conflict in their information about temporal rate, it is audition that dominates the percept. While this finding is not new, what is unique here is the use of a modality-independent procedure and thus the capability of assessing the magnitude of the auditory bias. In both experiments, the auditory bias of vision was approximately five times greater than the visual bias of audition. 
The fact that vision, the "inferior" modality in this situation, had some effect on audition is analogous to the finding that proprioception has a slight, but reliable, influence on vision in experiments in which vision and proprioception conflict about spatial location (e.g., Pick et al., 1969).

A question of interest now is whether it is possible to reduce, or perhaps even reverse, the strong influence of audition over vision in temporal rate perception. According to the "directed attention" hypothesis (e.g., Canon, 1971), this alteration of intersensory bias might be possible if the observer's attention could be drawn away from audition and onto the visual aspect of the repetitive visualauditory stimulus, just the opposite of the presumed "normal" distribution of attention. Ongoing research in our laboratory is investigating this possibility.

\section{REFERENCES}

Canon, L. K. (1970). Intermodality inconsistency of input and directed attention as determinants of the nature of adaptation. Journal of $E x$ perimental Psychology, 84, 141-147.

CANON, L. K. (1971). Directed attention and maladaptive "adaptation" to displacement of the visual field. Journal of Experimental Psychology, 88, 403-408.

Gebhard, J. W., \& Mowbray, G. H. (1959). On discriminating the rate of visual flicker and auditory flutter. American Journal of Psy chology, 72, 521-528.

Goldstone, S., Boardman, W. K., \& Lhamon, W. T. (1959). Intersensory comparisons of temporal judgments. Journal of Experimental Psychology, 57, 243-248.

Golstone, S., \& GoldFARB, J. L. (1963). Judgment of filled and unfilled durations: Intersensory factors. Perceptual \& Motor Skills, 17, 763-774.

HAY, J. C., PICK, H. L., JR., \& IKEDA, K. (1965). Visual capture produced by prism spectacles. Psychonomic Science, 2, 215-216.

HowARD, I. P., \& TEMPLETON, W. B. (1966). Human spatial orientation. New York: Wiley.

Kelso, J: A. S., Cook, E., Olson, M. E., \& Epstein, W. (1975). Allocation of attention and the locus of adaptation to displaced vision. Journal of Experimental Psychology: Human Perception \& Performance, 1, 237-245.

Myers, A. K. Cotton, B., \& Hilp, H. A. (1981). Matching the rate of concurrent tone bursts and light flashes as a function of flash surround luminance. Perception \& Psychophysics, 30, 33-38.

Pick, H. L., JR., Warren, D. H., \& Hay, J. C. (1969). Sensory conflict in judgments of spatial direction. Perception \& Psychophysics, 6, 203-205.
SHIPLEY, T. (1964). Auditory flutter-driving of visual flicker. Science, $145,1328-1330$.

UhLARIK, J. J., \& CANON, L. K. (1971). Influence of concurrent and terminal exposure conditions on the nature of perceptual adaptation. Journal of Experimental Psychology, 91, 233-239.

Warren, D. H., \& Cleaves, W. T. (1971). Visual-proprioceptive interaction under large amounts of conflict. Journal of Experimental Psychology, 90, 206-214.

WARREN, D. H., \& SCHMITT, T. J. (1978). On the plasticity of visualproprioceptive bias effects. Journal of Experimental Psychology: Human Perception \& Performance, 4, 302-310.

WELCH, R. B., \& WARREN, D. H. (1980). Immediate perceptual response to intersensory discrepancy. Psychological Bulletin, 88, 638-667.

\section{NOTES}

1. The existence of small, but statistically significant, proprioceptive bias of vision indicates that vision does not merely suppress proprioceptive information. This outcome also argues against the proposal that the observer becomes confused by the presence of the two forms of conflicting sensory information and, as a result, simply "chooses" to respond in terms of vision. A variation of the "confusion hypothesis" is that the observer's supposed bewilderment leads to haphazard responding from trial to trial. That this cannot be true is seen in the fact that the intertrial variability of the bisensory measures is no greater than that of the unisensory measures (Welch \& Warren, 1980).

2. Why this discrepancy occurs is unclear. Perhaps it is due to the well-established fact that both auditory durations and empty intervals bounded by auditory stimuli are perceived to be longer than physically equal visual durations and interstimulus intervals (e.g., Goldstone, Boardman, \& Lhamon, 1959; Goldstone \& Goldfarb, 1963). Thus, for example, if a 25 -msec-long auditory pulse and the interval between each pulse are both perceived as longer than a 25 -msec-long visual pulse and its associated interpulse interval, the perceived rate of the auditory stimulus might seem slower than that of the visual stimulus. Another possibility is that, because of the poor temporal acuity for repetitive visual, relative to auditory, stimuli, the former appear to be more "solid" (less empty space between pulses) than the latter and therefore are ascribed a higher perceived temporal rate.

3. Given the physical limitations of the experiment, it was not possible to verify that all subjects actually looked at the visual stimulus on all of the $A_{v}$ trials. If, instead, they had closed their eyes, then the present interpretation of the results is rendered a serious blow. However, during the postexperiment interview, none of the subjects reported that they had disobeyed this instruction. Thus, it seems reasonable to assume that any failure to keep the eyes open during the $A$ trials was, at most, a rare event and therefore not likely to have influenced the overall results of the experiment.

(Manuscript received August 24, 1984; revision accepted for publication April 2, 1986.) 